\title{
ON THE PERIODS OF GENERALIZED FIBONACCI RECURRENCES
}

\author{
RICHARD P. BRENT
}

\begin{abstract}
We give a simple condition for a linear recurrence $\left(\bmod 2^{w}\right)$ of degree $r$ to have the maximal possible period $2^{w-1}\left(2^{r}-1\right)$. It follows that the period is maximal in the cases of interest for pseudorandom number generation, i.e., for three-term linear recurrences defined by trinomials which are primitive $(\bmod 2)$ and of degree $r>2$. We consider the enumeration of certain exceptional polynomials which do not give maximal period, and list all such polynomials of degree less than 15 .
\end{abstract}

\section{INTRODUCTION}

The Fibonacci numbers satisfy a linear recurrence

$$
F_{n}=F_{n-1}+F_{n-2} \text {. }
$$

Generalized Fibonacci recurrences of the form

$$
x_{n}= \pm x_{n-s} \pm x_{n-r} \bmod 2^{w}
$$

are of interest because they are often used to generate pseudorandom numbers $[1,6,7,12,14,18]$. We assume throughout that $x_{0}, \ldots, x_{r-1}$ are given and not all even, and $w>0$ is a fixed exponent. Usually, $w$ is close to the wordlength of the (binary) computer used.

Apart from computational convenience, there is no reason to restrict attention to three-term recurrences of the special form (1). Thus, we consider a general linear recurrence

$$
q_{0} x_{n}+q_{1} x_{n+1}+\cdots+q_{r} x_{n+r}=0 \bmod 2^{w}
$$

defined by a polynomial $Q(t)=q_{0}+q_{1} t+\cdots+q_{r} t^{r}$ with integer coefficients and degree $r>0$. We assume throughout that $q_{0}$ and $q_{r}$ are odd. Because $q_{0}$ is odd, the sequence $\left(x_{n}\right)$ is reversible, i.e., $x_{n}$ is uniquely defined $\left(\bmod 2^{w}\right)$ by $x_{n+1}, \ldots, x_{n+r}$. Thus, $\left(x_{n}\right)$ is purely periodic [20].

Received by the editor May 4, 1992 and, in revised form, December 23, 1992.

1991 Mathematics Subject Classification. Primary 11Y55, 12E05, 05A15; Secondary 11-04, 11T06, 11T55, 12-04, 12E10, 65C10, 68R05.

Key words and phrases. Fibonacci sequence, generalized Fibonacci sequence, irreducible trinomial, linear recurrence, maximal period, periodic integer sequence, primitive trinomial, pseudorandom numbers. 
In the following we often work in a ring $\mathbf{Z}_{m}[t] / Q(t)$ of polynomials $(\bmod Q)$ whose coefficients are regarded as elements of $\mathbf{Z}_{m}$, the ring of integers $\bmod m$. For relations $A=B$ in $\mathbf{Z}_{m}[t] / Q(t)$ we use the notation

$$
A=B \bmod (m, Q) \text {. }
$$

It may be shown by induction on $n$ that, if $a_{n, 0}, \ldots, a_{n, r-1}$ are defined by

$$
t^{n}=\sum_{j=0}^{r-1} a_{n, j} t^{j} \bmod \left(2^{w}, Q(t)\right),
$$

then

$$
x_{n}=\sum_{j=0}^{r-1} a_{n, j} x_{j} \bmod 2^{w} .
$$

Also, the generating function

$$
G(t)=\sum_{n=0}^{\infty} x_{n} t^{n}
$$

is given by

$$
G(t)=\frac{P(t)}{\widetilde{Q}(t)} \bmod 2^{w}
$$

where

$$
P(t)=\sum_{k=0}^{r-1}\left(\sum_{j=0}^{k} q_{r+j-k} x_{j}\right) t^{k}
$$

is a polynomial of degree less than $r$, and

$$
\widetilde{Q}(t)=t^{r} Q(1 / t)=q_{0} t^{r}+q_{1} t^{r-1}+\cdots+q_{r}
$$

is the reverse of $Q$. In the literature, $\widetilde{Q}(t)$ is sometimes called the characteristic polynomial [5] or the associated polynomial [20] of the sequence. The use of generating functions is convenient and has been adopted by many earlier authors (e.g., Schur [16]). Ward [20] does not explicitly use generating functions, but his polynomial $U$ is the same as our $\widetilde{Q}$, and many of his results could be obtained via generating functions.

Let $\rho_{w}$ be the period of $t$ under multiplication $\bmod \left(2^{w}, Q(t)\right)$, i.e., $\rho_{w}$ is the least positive integer $\rho$ such that

$$
t^{\rho}=1 \bmod \left(2^{w}, Q(t)\right) .
$$

In the literature, $\rho_{w}$ is sometimes called the principal period [20] of the linear recurrence, sometimes simply the period [5]. For brevity we define $\lambda=\rho_{1}$.

If $Q(t)$ is irreducible in $\mathbf{Z}_{2}[t]$, then $Q(t)$ is a factor of $t^{2^{r}}-t$ (see, e.g., [19]), so $\lambda \mid 2^{r}-1$. We say that $Q(t)$ is primitive $(\bmod 2)$ if $\lambda=2^{r}-1$. Note that primitivity is a stronger condition than irreducibility, i.e., $Q(t)$ primitive 
implies that $Q(t)$ is irreducible ${ }^{1}$, but the converse is not generally true unless $2^{r}-1$ is prime. For example, the polynomial $1+t+t^{2}+t^{4}+t^{6}$ is irreducible, but not primitive, since it has $\lambda=21<2^{6}-1$. Tables of irreducible and primitive trinomials are available [5, 11, 15, 17, 21, 23, 24, 25].

In the following we usually assume that $Q(t)$ is irreducible. Our assumption that $q_{0}$ and $q_{r}$ are odd excludes the trivial case $Q(t)=t$, and implies that $\widetilde{Q}(t)$ is irreducible (or primitive) of degree $r$ if and only if the same is true of $Q(t)$.

We are interested in the period $p_{w}$ of the sequence $\left(x_{n}\right)$, i.e., the minimal positive $p$ such that

$$
x_{n+p}=x_{n}
$$

for all sufficiently large $n$. In fact, because of the reversibility of the sequence, (7) should hold for all $n$. The period is sometimes called the characteristic number of the sequence [20]. In general, the period depends on the initial values $x_{0}, \ldots, x_{r-1}$, but under our assumptions the period depends only on $Q(t)$, in fact $p_{w}=\rho_{w}$ (see Lemma 2).

It is known $[8,13,20]$ that $p_{w} \leq 2^{w-1} \lambda$, with equality holding for all $w>0$ if and only if it holds for $w=3$. The main aim of this paper is to give a simple necessary and sufficient condition for

$$
p_{w}=2^{w-1} \lambda \text {. }
$$

The result is stated in Theorem 2 in terms of a simple condition which we call "Condition $S$ " (see $\S 2$ ). In Theorem 3 we deduce that the period is maximal if $Q(t)$ is a primitive trinomial of degree greater than 2. Thus, in cases of practical interest for pseudorandom number generation, it is only necessary to verify that $Q(t)$ is primitive. This is particularly easy if $2^{r}-1$ is a Mersenne prime, because then a necessary and sufficient condition is

$$
t^{2^{r}}=t \bmod (2, Q(t)) \text {. }
$$

A word of caution is appropriate. Even when the period $p_{w}$ satisfies (8), it is not desirable to use a full cycle of $p_{w}$ numbers in applications requiring independent pseudorandom numbers. This is because only the most significant bit has the full period. If the bits are numbered from 1 (least significant) to $w$ (most significant), then bit $k$ has period $p_{k}$.

The basic results on linear recurrences modulo $m$ were obtained many years ago-see, for example, Ward [20]. However, our main results (Theorems 2 and 3 ) and the statement of "Condition S" (\$2) appear to be new.

\section{A CONDITION FOR MAXIMAL PERIOD}

The following lemma is a special case of Hensel's Lemma [8, 9, 22] and may be proved using an application of Newton's method for reciprocals [10].

Lemma 1. Suppose that $P(t) \bmod 2$ is invertible in $\mathrm{Z}_{2}[t] / Q(t)$. Then, for all $w \geq 1, P(t) \bmod 2^{w}$ is invertible in $\mathbf{Z}_{2^{w}}[t] / Q(t)$.

\footnotetext{
${ }^{1}$ For brevity we usually omit the "mod 2 " when saying that a polynomial is irreducible or primitive. Thus " $Q(t)$ is irreducible (resp. primitive)" means that $Q(t) \bmod 2$ is irreducible (resp. primitive) in $\mathbf{Z}_{2}[t]$.
} 
We now give a sufficient condition for the periods $p_{w}$ and $\rho_{w}$ to be the same.

Lemma 2. If $Q(t)$ is irreducible of degree $r$, and at least one of $x_{0}, \ldots, x_{r-1}$ is odd, then $p_{w}=\rho_{w}$.

Proof. For brevity we write $p=p_{w}$ and $\rho=\rho_{w}$. From (5),

$$
G(t)=\frac{R(t)}{1-t^{p}} \bmod 2^{w},
$$

where $R(t)$ has degree less than $p$. Thus, from (6),

$$
R(t) \widetilde{Q}(t)=\left(1-t^{p}\right) P(t) \bmod 2^{w} .
$$

$P(t) \bmod 2$ has degree less than $r$, but is not identically zero. Since $\widetilde{Q}(t) \bmod 2$ is irreducible of degree $r$, application of the extended Euclidean algorithm [8] to $P(t) \bmod 2$ and $\widetilde{Q}(t) \bmod 2$ constructs the inverse of $P(t) \bmod 2$ in $\mathbf{Z}_{2}[t] / \widetilde{Q}(t)$. Thus, Lemma 1 shows that $P(t) \bmod 2^{w}$ is invertible in $\mathbf{Z}_{2^{w}}[t] / \widetilde{Q}(t)$. It follows from (9) that

$$
t^{p}=1 \bmod \left(2^{w}, \widetilde{Q}(t)\right),
$$

and $\rho \mid p$. However, from (3) and (4), $p \mid \rho$. Thus, $p=\rho$.

As an example, consider $Q(t)=1-t+t^{2}$. We have $t^{3}=1 \bmod (2, Q(t))$, $t^{3}=-1 \bmod Q(t)$, and $t^{6}=1 \bmod Q(t)$, so

$$
\rho_{w}= \begin{cases}3 & \text { if } w=1 \\ 6 & \text { if } w>1\end{cases}
$$

It is easy to verify that $(10)$ gives the period $p_{w}$ of the corresponding recurrence

$$
x_{n}=x_{n-1}-x_{n-2} \quad \bmod 2^{w},
$$

provided $x_{0}$ and $x_{1}$ are not both even.

The assumption of irreducibility in Lemma 2 is significant. For example, consider $Q(t)=t^{2}-1$ and $w=1$, with initial values $x_{0}=x_{1}=1$. The recurrence is $x_{n}=x_{n-2} \bmod 2$, so $p_{1}=1$, but $\rho_{1}=2$. Here, $P(t)=1+t$ is a divisor of $\widetilde{Q}(t)=1-t^{2}$.

We now define a condition which must be satisfied by $Q( \pm t)$ if the period $p_{w}$ of the sequence $\left(x_{n}\right)$ is less than $2^{w-1} \lambda$ (see Theorem 2 for details). For given $Q(t)$ the condition can be checked in $O\left(r^{2}\right)$ operations, or in $O(r \log r)$ operations if the FFT is used to compute the convolutions in (11). Even the $O\left(r^{2}\right)$ algorithm is much faster than the method suggested by Marsaglia and Tsay [13], which involves forming high powers of $r \times r$ matrices $(\bmod 8)$, or the method of Knuth [8, ex. 3.2.2.11], which involves forming high powers in $\mathrm{Z}_{8}[t] / Q(t)$.

Condition S. Let $Q(t)=\sum_{j=0}^{r} q_{j} t^{j}$ be a polynomial of degree $r$. We say that $Q(t)$ satisfies Condition $\mathrm{S}$ if and only if

$$
Q(t)^{2}+Q(-t)^{2}=2 q_{r} Q\left(t^{2}\right) \bmod 8
$$


Lemma 3 gives an equivalent condition, which is more convenient for computational purposes. For another equivalent condition, see the remark following (22) in the proof of Theorem 1. The proof of Lemma 3 is straightforward, so is omitted.

Lemma 3. A polynomial $Q(t)$ of degree $r$ satisfies Condition $\mathrm{S}$ if and only if

$$
\sum_{\substack{j+k=2 m \\ 0 \leq j<k \leq r}} q_{j} q_{k}=\epsilon_{m} \bmod 2
$$

for $0 \leq m \leq r$, where

$$
\epsilon_{m}=\frac{q_{m}\left(q_{m}-q_{r}\right)}{2} .
$$

As an exercise, the reader may verify that the polynomial $Q(t)=1-t+t^{2}$ satisfies both the definition of Condition $S$ and the equivalent conditions of Lemma 3. For other examples, see Table 1.

For convenience we collect in Lemma 4 some results regarding arithmetic in the rings $\mathbf{Z}_{2^{w}}[t] / Q(t)$.

Lemma 4. Let $X(t)$ and $Y(t)$ be polynomials over $\mathbf{Z}$, and $Q(t)$ be as in $\S 1$. Then, for $w \geq 1$,

$$
X=Y \bmod \left(2^{w}, Q\right) \Rightarrow X^{2}=Y^{2} \bmod \left(2^{w+1}, Q\right) .
$$

Also, if $Q(t)$ is irreducible, then

$$
X^{2}=Y^{2} \bmod (2, Q) \Leftrightarrow X^{2}=Y^{2} \bmod (4, Q)
$$

and

$$
X^{2}=Y^{2} \bmod (8, Q) \Leftrightarrow X= \pm Y \bmod (4, Q) .
$$

Proof. If $X=Y \bmod \left(2^{w}, Q\right)$, then $X=Y+2^{w} R \bmod Q$ for some polynomial $R(t)$ in $\mathrm{Z}[t]$. Thus, $X^{2}=Y^{2}+2^{w+1} R\left(Y+2^{w-1} R\right) \bmod Q$, and (13) follows.

If $Q(t)$ is irreducible and $X^{2}=Y^{2} \bmod (2, Q)$, then $(X-Y)^{2}=0 \bmod (2, Q)$. Since $Q$ is irreducible, it follows that $X=Y \bmod (2, Q)$. Thus, from (13), $X^{2}=Y^{2} \bmod (4, Q)$, and (14) follows.

Finally, if $Q$ is irreducible and $X^{2}=Y^{2} \bmod (8, Q)$ then, as in the proof of (14), we obtain $X=Y \bmod (2, Q)$, so $X=Y+2 R \bmod Q$, where $R(t)$ is some polynomial in $\mathrm{Z}[t]$. Thus, $4 R(Y+R)=0 \bmod (8, Q)$, i.e., $R(Y+R)=$ $0 \bmod (2, Q)$. Since $Q$ is irreducible, either $R=0 \bmod (2, Q)$ or $Y+R=$ $0 \bmod (2, Q)$. In the former case, $X=Y \bmod (4, Q)$, and in the latter case, $X=-Y \bmod (4, Q)$. Thus, $X= \pm Y \bmod (4, Q)$. The implication in the other direction follows from (13). This establishes (15).

The following result is the key to the proof of Theorem 2 . There is no obvious generalization to odd moduli. Recall that $\lambda=\rho_{1}$. 
Theorem 1. Let $Q(t) \bmod 2$ be irreducible in $\mathrm{Z}_{2}[t]$. Then

$$
t^{\lambda}=-1 \bmod (4, Q(t))
$$

if and only if $Q(t)$ satisfies Condition $\mathrm{S}$, and

$$
t^{\lambda}=1 \bmod (4, Q(t))
$$

if and only if $Q(-t)$ satisfies Condition $\mathrm{S}$.

Proof. Let

$$
V(t)=\sum_{j=0}^{\lfloor r / 2\rfloor} q_{2 j} t^{j}, \quad W(t)=\sum_{j=0}^{\lfloor(r-1) / 2\rfloor} q_{2 j+1} t^{j},
$$

so $Q(t)$ splits into even and odd parts:

$$
Q(t)=V\left(t^{2}\right)+t W\left(t^{2}\right) .
$$

By the definition of $\lambda$, we have $t=t^{\lambda+1} \bmod (2, Q(t))$, so

$$
V\left(t^{2}\right)=t^{\lambda+1} W\left(t^{2}\right) \bmod (2, Q(t)) .
$$

Because $X\left(t^{2}\right)=X(t)^{2} \bmod 2$ for any polynomial $X(t)$ in $\mathbf{Z}[t]$, equation (17) may be written as

$$
V(t)^{2}=t^{\lambda+1} W(t)^{2} \bmod (2, Q(t)) .
$$

Since $\lambda$ is a divisor of $2^{r}-1$, it is odd, so $t^{\lambda+1}$ is a square. Thus, from (14),

$$
V(t)^{2}=t^{\lambda+1} W(t)^{2} \bmod (4, Q(t)) .
$$

Also, since $V(t)=V(-t) \bmod 2$ and $W(t)=W(-t) \bmod 2$, we have

$$
V(-t)^{2}=t^{\lambda+1} W(-t)^{2} \bmod (4, Q(t)) .
$$

To prove the first half of the theorem, suppose that

$$
t^{\lambda}=-1 \bmod (4, Q(t)) \text {. }
$$

Thus, from (18),

$$
V(t)^{2}+t W(t)^{2}=0 \bmod (4, Q(t)) .
$$

It follows that

$$
V(t)^{2}+t W(t)^{2}-q_{r} Q(t)=0 \bmod (4, Q) .
$$

However, the left side of (21) is a polynomial of degree less than $r$. Hence,

$$
V(t)^{2}+t W(t)^{2}-q_{r} Q(t)=0 \bmod 4 .
$$

Replace $t$ by $t^{2}$ in the identity (22). From (16), the result is easily seen to be equivalent to $Q(t)$ satisfying Condition $\mathrm{S}$. 
To prove the converse, suppose that $Q(t)$ satisfies Condition S. Reversing our argument, we see that (20) holds. Thus, from (18),

$$
\left(t^{\lambda+1}+t\right) W(t)^{2}=0 \bmod (4, Q(t)) .
$$

Now $W(t)$ has degree less than $r$, and $W(t) \neq 0 \bmod 2$, because otherwise, using (16), $Q(t)=V(t)^{2} \bmod 2$ would contradict the irreducibility of $Q(t)$. It follows that $W(t) \bmod 2$ is invertible in $Z_{2}[t] / Q(t)$. From Lemma $1, W(t) \bmod 4$ is invertible in $\mathbf{Z}_{4}[t] / Q(t)$, and we obtain

$$
t^{\lambda+1}+t=0 \bmod (4, Q(t)) .
$$

Since $Q(t) \neq t \bmod 2$, we can divide by $t$ to obtain

$$
t^{\lambda}=-1 \bmod (4, Q(t)) \text {. }
$$

This completes the proof of the first half of the theorem.

The proof of the second half is similar, with appropriate changes of sign. Suppose that

$$
t^{\lambda}=1 \bmod (4, Q(t))
$$

From (19),

$$
V(-t)^{2}=t W(-t)^{2} \bmod (4, Q(t)) .
$$

Thus, instead of (22) we obtain

$$
V(-t)^{2}-t W(-t)^{2}-(-1)^{r} q_{r} Q(t)=0 \bmod 4 .
$$

Replace $t$ by $-t^{2}$ in the identity (24). The result is equivalent to $Q(-t)$ satisfying Condition $\mathrm{S}$. The converse also applies: if $Q(-t)$ satisfies Condition $\mathrm{S}$ then, by reversing our argument and using irreducibility of $Q(t)$, we find that (23) holds.

We are now ready to state Theorem 2 , which relates the period of the sequence $\left(x_{n}\right)$ to Condition S. In view of Theorem 1, Theorem 2 is implicit in Ward [20, p. 628]. More precisely, Ward's case $T>1$ corresponds to $Q(-t)$ satisfying Condition S, while Ward's case $(T=1, K(x)=1 \bmod 2)$ corresponds to $Q(t)$ satisfying Condition S. However, Ward's exposition is complicated by consideration of odd prime power moduli (see for example his Theorem 13.1), so we give an independent proof.

Theorem 2. Let $Q(t)$ be irreducible and define a linear recurrence by (2), with at least one of $x_{0}, \ldots, x_{r-1}$ odd. Then the sequence $\left(x_{n}\right)$ has period

$$
p_{w} \leq 2^{w-2} \lambda
$$

for all $w \geq 2$ if $Q(-t)$ satisfies Condition $\mathrm{S}$,

$$
p_{w} \leq 2^{w-2} \lambda
$$

for all $w \geq 3$ if $Q(t)$ satisfies Condition $\mathrm{S}$, and

$$
p_{w}=2^{w-1} \lambda
$$

for all $w \geq 1$ if and only if neither $Q(t)$ nor $Q(-t)$ satisfies Condition $\mathrm{S}$. 
Proof. From Lemma 2, $p_{w}=\rho_{w}$ is the order of $t \bmod \left(2^{w}, Q(t)\right)$. If $Q(-t)$ satisfies Condition $\mathrm{S}$, then, from Theorem 1 ,

$$
t^{\lambda}=1 \bmod (4, Q(t)) .
$$

By (13), it follows by induction on $w$ that

$$
t^{2^{w-2} \lambda}=1 \bmod \left(2^{w}, Q(t)\right)
$$

for all $w \geq 2$. This proves the first part of the theorem. The second part is similar, so it only remains to prove the third part.

Suppose that $\rho_{w}=2^{w-1} \lambda$ for all $w>0$. In particular, for $w=3$ we have period $\rho_{3}=4 \lambda$. Thus,

and, from (15),

$$
t^{2 \lambda} \neq 1 \quad \bmod (8, Q(t))
$$

$$
t^{\lambda} \neq \pm 1 \bmod (4, Q(t)) .
$$

From Theorem 1, neither $Q(t)$ nor $Q(-t)$ can satisfy Condition S, or we would obtain a contradiction to (25).

Conversely, if neither $Q(t)$ nor $Q(-t)$ satisfies Condition $\mathrm{S}$, then we show by induction on $w$ that

$$
t^{2^{w-1} \lambda}=1+2^{w} R_{w} \bmod Q(t),
$$

where

$$
R_{w} \neq 0 \bmod (2, Q(t)),
$$

for all $w \geq 1$. Certainly,

$$
t^{\lambda}=1 \bmod (2, Q(t)),
$$

but, from Theorem 1,

$$
t^{\lambda} \neq 1 \quad \bmod (4, Q(t)),
$$

so (26) and (27) hold for $w=1$. Defining

$$
R_{w}=R_{w-1}\left(1+2^{w-2} R_{w-1}\right)
$$

for $w \geq 2$, we see that (26) holds for all $w \geq 1$. It remains to prove (27) for $w>1$.

For $w=2$, inequality (27) follows from Theorem 1 and (15), as $t^{\lambda} \neq$ $\pm 1 \bmod (4, Q(t))$ implies $t^{2 \lambda} \neq 1 \bmod (8, Q(t))$. For $w>2$, the inequality (27) follows by induction from (28), since $2^{w-2}$ is even. It follows that $\rho_{w}=$ $2^{w-1} \lambda$ for all $w \geq 1$.

\section{Primitive trinomials}

In this section we consider a case of interest because of its applications to pseudorandom number generation:

$$
Q(t)=q_{0}+q_{s} t^{s}+q_{r} t^{r}
$$

is a trinomial $(r>s>0)$. Theorem 3 shows that the period is always maximal in cases of practical interest. The condition $r>2$ is necessary, as the example $Q(t)=1-t+t^{2}$ of $\S 2$ shows. 
Theorem 3. Let $Q(t)=q_{0}+q_{s} t^{s}+q_{r} t^{r}$ be a primitive trinomial of degree $r>2$. Then the sequence $\left(x_{n}\right)$ defined by (2), with at least one of $x_{0}, \ldots, x_{r-1}$ odd, has period $p_{w}=2^{w-1}\left(2^{r}-1\right)$.

Proof. From Theorem 2 it is sufficient to show that $Q(t)$ does not satisfy Condition $\mathrm{S}$. (Since $Q(-t)$ is also a trinomial, the same argument shows that $Q(-t)$ does not satisfy Condition S.)

Suppose, by way of contradiction, that $Q(t)$ satisfies Condition S. We use the formulation of Condition S given in Lemma 3. Since $Q(t)$ is irreducible, we have $q_{0}=q_{s}=q_{r}=1 \bmod 2$. If $s$ is even, say $s=2 m$, then

$$
\sum_{\substack{j+k=2 m \\ 0 \leq j<k \leq r}} q_{j} q_{k}=q_{0} q_{s}=1 \bmod 2,
$$

so $\epsilon_{m} \neq 0$, and (12) implies that $q_{m} \neq 0$. Since $0<m<s<r$, this contradicts the assumption that $Q(t)$ is a trinomial. Hence, $s$ must be odd.

If $r$ is odd then $r+s$ is even, and a similar argument shows that $q_{(r+s) / 2} \neq 0$, contradicting the assumption that $Q(t)$ is a trinomial. Hence, $r$ must be even.

Taking $m=r / 2$, we see that $\epsilon_{m} \neq 0$, so $q_{m} \neq 0$. This is only possible if $m=s$, so $Q(t)=t^{2 s}+t^{s}+1 \bmod 2$. In this case, $t^{3 s}=1 \bmod (2, Q(t))$. Now $r=2 s>2$, so $3 s<2^{r}-1$, and $Q(t)$ cannot be primitive. This contradiction completes the proof.

A minor modification of the proof of Theorem 3 gives:

Theorem 4. Let $Q(t)=q_{0}+q_{s} t^{s}+q_{r} t^{r}$ be an irreducible trinomial of degree $r \neq 2 s$. Then the sequence $\left(x_{n}\right)$ defined by (2), with at least one of $x_{0}, \ldots, x_{r-1}$ odd, has period $p_{w}=2^{w-1} \lambda$.

As mentioned above, it is easy to find primitive trinomials of very high degree $r$ if $2^{r}-1$ is a Mersenne prime. Zierler [24] gives examples with $r \leq 9689$, and we found two examples with higher degree: $t^{19937}+t^{9842}+1$ and $t^{23209}+$ $t^{9739}+1$. These and other examples with $r \leq 44497$ were found independently by Kurita and Matsumoto [11]. Such primitive trinomials provide the basis for fast random number generators with extremely long periods and good statistical properties [3]. In general, random number generators with larger $r$ have better statistical properties than those with smaller $r$, and generators with small $r$ should be avoided [3, 4].

\section{EXCEPTIONAL POLYNOMIALS}

We say that a polynomial $Q(t)$ of degree $r>1$ is exceptional if conditions E1-E3 hold, and is a candidate if conditions E2-E3 hold:

E1. $Q(t) \bmod 2$ is primitive.

E2. $Q(t)$ has coefficients $q_{j} \in\{0,-1,+1\}$, and $q_{0}=q_{r}=1$.

E3. $Q(t)$ satisfies Condition $\mathrm{S}$.

If $Q(t)$ is exceptional then, by Theorem 2, $Q(t)$ and $Q(-t)$ define linear recurrences $\left(\bmod 2^{w}\right)$ which have less than the maximal period for all $w>2$. In Table 1 we list the exceptional polynomials $Q(t)$ of degree $r \leq 14$. If $Q(t)$ is exceptional, then so is $\widetilde{Q}(t)$. Thus, we only list one of these in Table 1 . 
TABLE 1. Exceptional polynomials of degree $r \leq 14$

\begin{tabular}{|c|c|}
\hline$r$ & $Q(t)$ \\
\hline 2 & $1-t+t^{2}$ \\
\hline 5 & $1-t-t^{2}+t^{4}+t^{5}$ \\
\hline & $1-t+t^{2}+t^{3}-t^{4}-t^{6}+t^{9}$ \\
9 & $1-t+t^{2}-t^{3}-t^{4}+t^{8}+t^{9}$ \\
& $1-t+t^{2}-t^{3}-t^{4}-t^{5}+t^{6}+t^{8}+t^{9}$ \\
\hline 10 & $1-t+t^{2}+t^{3}+t^{4}+t^{6}-t^{7}+t^{9}+t^{10}$ \\
\hline 11 & $1-t+t^{2}-t^{3}-t^{4}+t^{5}+t^{6}-t^{8}+t^{11}$ \\
\hline 12 & $1-t+t^{2}-t^{3}-t^{4}-t^{8}+t^{9}+t^{11}+t^{12}$ \\
\hline \multirow{4}{*}{13} & $1-t+t^{2}-t^{3}+t^{4}-t^{5}-t^{6}+t^{12}+t^{13}$ \\
& $1-t+t^{2}-t^{3}+t^{4}-t^{5}-t^{6}-t^{7}+t^{8}+t^{12}+t^{13}$ \\
& $1-t-t^{2}-t^{4}-t^{6}+t^{7}-t^{8}+t^{9}+t^{10}+t^{12}+t^{13}$ \\
& $1-t+t^{2}+t^{3}+t^{4}+t^{5}+t^{7}+t^{9}-t^{11}-t^{12}+t^{13}$ \\
& $1-t+t^{2}+t^{3}+t^{4}+t^{5}-t^{8}-t^{9}-t^{11}-t^{12}+t^{13}$ \\
\hline \multirow{4}{*}{14} & $1-t+t^{2}+t^{3}-t^{4}-t^{6}-t^{7}+t^{8}+t^{9}-t^{11}+t^{14}$ \\
& $1+t+t^{3}-t^{4}-t^{5}+t^{6}+t^{7}+t^{8}+t^{9}-t^{11}+t^{14}$ \\
& $1-t-t^{2}+t^{3}-t^{5}+t^{6}+t^{7}-t^{8}-t^{9}+t^{13}+t^{14}$ \\
& $1-t-t^{2}-t^{3}-t^{5}+t^{7}+t^{9}+t^{10}-t^{11}+t^{13}+t^{14}$ \\
& $1-t-t^{2}+t^{4}-t^{6}+t^{8}+t^{9}+t^{10}+t^{11}+t^{13}+t^{14}$ \\
\hline
\end{tabular}

Only the coefficients of $Q(t) \bmod 4$ are relevant to Condition $\mathrm{S}$. If condition E2 is relaxed to allow coefficients equal to 2, then, by Lemma 3, there is one such $Q(t)$ corresponding to each primitive polynomial in $\mathbf{Z}_{2}[t]$. With condition E2 as stated, the number of these $Q(t)$ is considerably reduced.

It is interesting to consider strengthening condition E2 by asking for certain patterns in the signs of the coefficients. For example, we might ask for polynomials $Q(t)$ with all coefficients $q_{j} \in\{0,1\}$, or for all coefficients of $\pm Q(-t)$ to be in $\{0,1\}$. There are candidates satisfying these conditions, but we have not found any which are also exceptional, apart from the trivial $Q(t)=1-t+t^{2}$. It is possible for an exceptional polynomial to have $(-1)^{j} q_{j} \geq 0$ for $0 \leq j<r$. The only example for $2<r \leq 44$ is

$$
Q(t)=1-t+t^{2}-t^{5}+t^{6}+t^{8}-t^{9}+t^{10}+t^{12}-t^{13}+t^{16}+t^{18}+t^{21} .
$$

Observe that $Q(-t)$ defines a linear recurrence with nonnegative coefficients

$$
\begin{aligned}
x_{n+21}= & x_{n}+x_{n+1}+x_{n+2}+x_{n+5}+x_{n+6}+x_{n+8} \\
& +x_{n+9}+x_{n+10}+x_{n+12}+x_{n+13}+x_{n+16}+x_{n+18},
\end{aligned}
$$

which has period $p_{2}=p_{1}=2^{21}-1$ when considered $\bmod 2$ or $\bmod 4$.

The number $\nu(r)$ of exceptional $Q(t)$ (counting only one of $Q(t), \widetilde{Q}(t))$ is given in Table 2. The term "exceptional" is justified as $\nu(r)$ appears to be a much more slowly growing function of $r$ than the number [5]

$$
\lambda_{2}(r)=\varphi\left(2^{r}-1\right) / r
$$


TABLE 2. Number of exceptional polynomials

\begin{tabular}{|c|c|c||c|c|c||c|c|c||c|c|c|}
\hline$r$ & $\nu(r)$ & $\bar{\nu}(r)$ & $r$ & $\nu(r)$ & $\bar{\nu}(r)$ & $r$ & $\nu(r)$ & $\bar{\nu}(r)$ & $r$ & $\nu(r)$ & $\bar{\nu}(r)$ \\
\hline 1 & 0 & 0 & 11 & 1 & 0.13 & 21 & 79 & 0.3923 & 31 & 4380 & 0.4721 \\
2 & 1 & 1.78 & 12 & 1 & 0.22 & 22 & 94 & 0.4390 & 32 & 3125 & 0.4636 \\
3 & 0 & 0 & 13 & 5 & 0.33 & 23 & 231 & 0.4837 & 33 & 7232 & 0.4549 \\
4 & 0 & 0 & 14 & 5 & 0.37 & 24 & 129 & 0.4650 & 34 & 8862 & 0.4656 \\
5 & 1 & 0.70 & 15 & 15 & 0.62 & 25 & 428 & 0.4388 & 35 & 18870 & 0.4792 \\
6 & 0 & 0 & 16 & 12 & 0.58 & 26 & 448 & 0.4615 & 36 & 10516 & 0.4560 \\
7 & 0 & 0 & 17 & 26 & 0.45 & 27 & 883 & 0.4964 & 37 & 40082 & 0.4547 \\
8 & 0 & 0 & 18 & 18 & 0.41 & 28 & 635 & 0.4218 & 38 & 39858 & 0.4623 \\
9 & 3 & 0.83 & 19 & 62 & 0.53 & 29 & 1933 & 0.4410 & 39 & 75370 & 0.4712 \\
10 & 1 & 0.30 & 20 & 34 & 0.45 & 30 & 1470 & 0.4619 & 40 & 54758 & 0.4598 \\
\hline
\end{tabular}

of primitive polynomials of degree $r$ in $\mathbf{Z}_{2}[t]$ (where $\varphi$ is Euler's totient function) or the total number of polynomials of degree $r$ with coefficients in $\{0,-1,+1\}$. A heuristic argument suggests that the number $\kappa(r)$ of candidates should grow like $(3 / 2)^{r}$ and that $\nu(r)$ should grow like $(3 / 4)^{r} \lambda_{2}(r)$. The argument is as follows:

There are $2^{r-1}$ polynomials $\bar{Q}(t)$ of degree $r$ with coefficients in $\{0,1\}$, satisfying $\bar{q}_{0}=\bar{q}_{r}=1$. Randomly select such a $\bar{Q}(t)$, and compute $\epsilon_{0}, \epsilon_{1}, \ldots, \epsilon_{r}$ from

$$
\sum_{\substack{j+k=2 m \\ 0 \leq j<k \leq r}} \bar{q}_{j} \bar{q}_{k}=\epsilon_{m} \bmod 2 .
$$

Extend $\bar{Q}(t)$ to a polynomial $Q(t)$ with coefficients $q_{m} \in\{-1,0,1,2\}$ such that $\bar{q}_{m}=q_{m} \bmod 2$ and (12) is satisfied for $0 \leq m \leq r$. The (unique) mapping is given by $q_{m}=\bar{q}_{m}+2 \epsilon_{m} \bmod 4$. It is easy to see that $q_{0}=q_{r}=1$. If we assume that, for $1 \leq m<r$, each $q_{m}$ has independent probability $1 / 4$ of assuming the "forbidden" value 2 , then the probability that $Q(t)$ is a candidate is $(3 / 4)^{r-1}$. Thus,

$$
\kappa(r) \simeq(3 / 2)^{r-1} .
$$

The probability that a randomly chosen $\bar{Q}(t)$ with $\bar{q}_{0}=\bar{q}_{r}=1$ is primitive is just $\lambda_{2}(r) / 2^{r-1}$. If there is the same probability that a randomly chosen candidate is primitive, then the number of primitive candidates should be $(3 / 4)^{r-1} \lambda_{2}(r)$, and $\nu(r)$ should be half this number.

The argument is not strictly correct. For example, it gives a positive probability that $q_{1}=0, q_{2}=1$, but this never occurs for $r>2$. However, the argument does appear to predict the correct order of magnitude of $\kappa(r)$ and $\nu(r)$. In Table 2 we give

$$
\bar{\nu}(r)=\frac{\nu(r)}{(3 / 4)^{r} \lambda_{2}(r)} ;
$$

the numerical evidence suggests that $\bar{\nu}(r)$ converges to a positive constant $\bar{\nu}(\infty)$ as $r \rightarrow \infty$. However, $\bar{\nu}(\infty)$ is less than the value $2 / 3$ predicted by the heuristic argument. Our best estimate (obtained from a separate computation which 
gives faster convergence) is

$$
\bar{\nu}(\infty)=0.45882 \pm 0.00002 \text {. }
$$

The computation of Table 2 took 166 hours on a VaxStation 3100 . We outline the method used. It is easy to check if a candidate polynomial is exceptional [8]. A straightforward method of enumerating all candidate polynomials of degree $r$ is to associate a polynomial $Q(t)$ such that $q_{0}=q_{r}=1$ with an $(r-1)$ bit binary number $N=b_{1} \cdots b_{r-1}$, where $b_{j}=q_{j} \bmod 2$. For each such $N$, compute $\epsilon_{0}, \ldots, \epsilon_{r}$ from (11). Now (12) defines $q_{0}, \ldots, q_{r} \bmod 4$. If there is an index $m$ such that $\epsilon_{m}=1 \bmod 2$ but $q_{m}=0 \bmod 2$, then (12) shows that $q_{m}=2 \bmod 4$, contradicting condition E2. The straightforward enumeration has complexity $\Omega\left(2^{r}\right)$, but this can be reduced by two devices:

A. If (12) shows that $q_{m}=2 \bmod 4$ for some $m<r / 2$, we may use the fact that $\epsilon_{m}$ in (11) depends only on $q_{0}, \ldots, q_{2 m}$ to skip over a block of $2^{r-2 m-1}$ numbers $N$. By an argument similar to the heuristic argument for the order of magnitude of $\nu(r)$, with support from empirical evidence for $r \leq 40$, we conjecture that this device reduces the complexity of the enumeration to

$$
O\left(r^{2} 2^{r}(3 / 4)^{r / 2}\right)=O\left(r^{2} 3^{r / 2}\right) .
$$

B. Fix $s, 0 \leq s<r$. Since $\epsilon_{r-m}$ in (11) depends only on $q_{r-2 m}, \ldots, q_{r}$, we can tabulate those low-order bits $b_{r-s} \cdots b_{r-1}$ which do not necessarily lead to condition E2 being violated for some $q_{r-m}, 2 m \leq s$. In the enumeration we need only consider $N$ with low-order bits in the table. We conjecture that this reduces the complexity of the enumeration to

$$
O\left(r^{2} 2^{r}(3 / 4)^{s / 2}\right)=O\left(r^{2} 2^{r-s} 3^{s / 2}\right),
$$

provided care is taken to generate the table efficiently.

The two devices can be combined, but they are not independent. The complexity of the combination is conjectured to be

$$
O\left(r^{2} 2^{r}(3 / 4)^{(6 r+5 s) / 12}\right)=O\left(r^{2} 3^{r / 2}(3 / 4)^{5 s / 12}\right),
$$

where the exponent $5 s / 12$ (instead of $s / 2$ ) reflects the lack of independence. In the computation of Table 2 we used $s \leq 22$ because of memory constraints. The table size is $O\left(s 3^{s / 2}\right)$ bits, if the table is stored as a list to take advantage of sparsity.

Note added in proof. Examples of primitive trinomials with $r \leq 132049$ were recently found by Heringa, Blöte and Compagner, Internat. J. Modern Phys. C 3 (1992), 561-564.

\section{ACKNOWLEDGMENTS}

We thank a referee for pointing out an error in the formulation of Lemma 2 given in [2], and for providing references to the classical literature. Richard Walker's assistance with $\mathcal{A}_{\mathcal{M}} \mathcal{S}$-LATEX was invaluable. The ANU Supercomputer Facility provided time on a Fujitsu VP 2200/10 for the discovery of the primitive trinomials mentioned at the end of $\S 3$. 


\section{BIBLIOGRAPHY}

1. S. L. Anderson, Random number generators on vector supercomputers and other advanced architectures, SIAM Rev. 32 (1990), 221-251.

2. R. P. Brent, On the periods of generalized Fibonacci recurrences, Technical Report TR-CS92-03, Computer Sciences Laboratory, Australian National University, Canberra, March 1992.

3. $ـ$ Uniform random number generators for supercomputers, Proc. Fifth Australian Supercomputer Conference, Melbourne, Dec. 1992, pp. 95-104.

4. A. M. Ferrenberg, D. P. Landau, and Y. J. Wong, Monte Carlo simulations: Hidden errors from "good" random number generators, Phys. Rev. Lett. 69 (1992), 3382-3384.

5. S. W. Golomb, Shift register sequences, Holden-Day, San Francisco, 1967.

6. B. F. Green, J. E. K. Smith, and L. Klem, Empirical tests of an additive random number generator, J. Assoc. Comput. Mach. 6 (1959), 527-537.

7. F. James, A review of pseudorandom number generators, Comput. Phys. Comm. 60 (1990), 329-344.

8. D. E. Knuth, The art of computer programming, Volume 2: Seminumerical algorithms (2nd ed.), Addison-Wesley, Menlo Park, CA, 1981.

9. E. V. Krishnamurthy, Error-free polynomial matrix computations, Chapter 4, SpringerVerlag, New York, 1985.

10. H. T. Kung, On computing reciprocals of power series, Numer. Math. 22 (1974), 341-348.

11. Y. Kurita and M. Matsumoto, Primitive $t$-nomials $(t=3,5)$ over $\mathrm{GF}(2)$ whose degree is a Mersenne exponent $\leq 44497$, Math. Comp. 56 (1991), 817-821.

12. G. Marsaglia, A current view of random number generators, Computer Science and Statistics: The Interface (edited by L. Billard), Elsevier Science Publishers B. V. (North-Holland), 1985, 3-10.

13. G. Marsaglia and L. H. Tsay, Matrices and the structure of random number sequences, Linear Algebra Appl. 67 (1985), 147-156.

14. J. F. Reiser, Analysis of additive random number generators, $\mathrm{Ph}$. D. thesis, Department of Computer Science, Stanford University, Stanford, CA, 1977. Also Technical Report STANCS-77-601.

15. E. R. Rodemich and H. Rumsey, Jr., Primitive trinomials of high degree, Math. Comp. 22 (1968), 863-865.

16. I. Schur, Ganzzahlige Potenzreihen und linear rekurrente Zahlenfolgen, Issai Schur Gesammelte Abhandlungen, Band 3, Springer-Verlag, Berlin, 1973, pp. 400-421.

17. W. Stahnke, Primitive binary polynomials, Math. Comp. 27 (1973), 977-980.

18. R. C. Tausworthe, Random numbers generated by linear recurrence modulo two, Math. Comp. 19 (1965), 201-209.

19. B. L. van der Waerden, Algebra, Vol. 1, Chapter 7 (English transl. by Fred Blum, 5th ed.), Ungar, New York, 1953.

20. M. Ward, The arithmetical theory of linear recurring series, Trans. Amer. Math. Soc. 35 (1933), 600-628.

21. E. J. Watson, Primitive polynomials $(\bmod 2)$, Math. Comp. $16(1962), 368-369$.

22. H. Zassenhaus, On Hensel factorization, J. Number Theory 1 (1969), 291-311.

23. N. Zierler and J. Brillhart, On primitive trinomials $(\bmod 2)$, Inform. and Control $13(1968)$, 541-554. Also part II, ibid. 14 (1969), 566-569.

24. N. Zierler, Primitive trinomials whose degree is a Mersenne exponent, Inform. and Control 15 (1969), 67-69.

25. $\_$, On $x^{n}+x+1$ over GF (2), Inform. and Control 16 (1970), 502-505.

Computer Sciences laboratory, Australian National University, Canberra, ACT 0200, Australia

E-mail address: rpbecslab.anu.edu.au 